\title{
Homology cobordism and classical knot invariants
}

\author{
Christian Bohr and Ronnie Lee
}

\begin{abstract}
In this paper, we define and investigate $\mathbb{Z}_{2}$-homology cobordism invariants of $\mathbb{Z}_{2^{-}}$ homology 3-spheres which turn out to be related to classical invariants of knots. As an application, we show that many lens spaces have infinite order in the $\mathbb{Z}_{2}$-homology cobordism group and we prove a lower bound for the slice genus of a knot on which integral surgery yields a given $\mathbb{Z}_{2}$-homology sphere. We also give some new examples of 3-manifolds which cannot be obtained by integral surgery on a knot.
\end{abstract}

Mathematics Subject Classification (2000). 57M27, 57M25.

Keywords. Homology 3-spheres, homology cobordism, slice genus.

In recent years, gauge theoretical tools and new results in 4-dimensional topology have successfully been used to study the structure of the integral homology cobordism group. It is for instance a consequence of Donaldson's famous theorem about the intersection forms of smooth 4-manifolds that the Poincaré homology sphere $\Sigma(2,3,5)$ has infinite order in this group, and M. Furuta found a family of Brieskorn spheres which generates a subgroup of infinite rank [12]. Recently N. Saveliev [22] showed, using the $w$-invariant introduced in [11], that a Brieskorn sphere with non-trivial Rokhlin invariant has infinite order in the integral homology cobordism group.

However, many 3-manifolds arising naturally in knot theory, for instance double coverings of the 3 -sphere branched along knots, are not integral homology spheres, but still $\mathbb{Z}_{2}$-homology spheres. As in the case of integral homology spheres, the set of $\mathbb{Z}_{2}$-homology spheres modulo the $\mathbb{Z}_{2}$-homology cobordism relation forms a group, the so called $\mathbb{Z}_{2}$-homology cobordism group $\Theta_{\mathbb{Z}_{2}}^{3}$. To study this group, we introduce, based on Furuta's result on the intersection forms of smooth 4dimensional spin manifolds [13], two invariants of $\mathbb{Z}_{2}$-homology spheres which turn out to be in fact invariants of the cobordism class (see Theorem 1). Exploiting that these invariants are closely related to classical knot invariants like signature and slice genus, we prove estimates for them in the case of lens spaces, which enables us to exhibit many examples of lens spaces which have infinite order in

\footnotetext{
The first author gratefully acknowledges support from the Deutsche Forschungsgemeinschaft.
} 
the $\mathbb{Z}_{2}$-homology cobordism group, and we determine the slice genera of certain Montesinos knots.

A second relation between knot theory and cobordism classes of $\mathbb{Z}_{2}$-homology spheres is provided by the simple fact that surgery along a knot with odd framing produces a $\mathbb{Z}_{2}$-homology sphere. In this case, our invariants can again be related to the slice genus of the knot. Using this, we prove a lower bound for the slice genera of knots on which integral surgery yields a given $\mathbb{Z}_{2}$-homology sphere. We also give new examples of 3 -manifolds which cannot be obtained by integral surgery on a knot.

\section{Bordism invariants of $\mathbb{Z}_{2}$-homology spheres}

Recall that a closed connected and oriented 3 -manifold $\Sigma$ is called a $\mathbb{Z}_{2}$-homology sphere if $H_{*}\left(\Sigma ; \mathbb{Z}_{2}\right)=H_{*}\left(S^{3} ; \mathbb{Z}_{2}\right)$. Two $\mathbb{Z}_{2}$-homology spheres $\Sigma_{1}, \Sigma_{2}$ are called $\mathbb{Z}_{2^{-}}$ homology cobordant if there exists a smooth 4-dimensional manifold $W$ with $\partial W=$ $\Sigma_{1}-\Sigma_{2}$ such that the inclusions $\Sigma_{i} \rightarrow W$ induce isomorphisms $H_{*}\left(\Sigma_{i} ; \mathbb{Z}_{2}\right) \rightarrow$ $H_{*}\left(W ; \mathbb{Z}_{2}\right)$. The set of $\mathbb{Z}_{2}$-homology cobordism classes of $\mathbb{Z}_{2}$-homology spheres forms a group, the so called $\mathbb{Z}_{2}$-homology cobordism group, which we denote by $\Theta_{\mathbb{Z}_{2}}^{3}$. Addition in this group is given by taking the connected sum, and the zero element is the equivalence class of the 3 -sphere.

If $\Sigma$ is a $\mathbb{Z}_{2}$-homology sphere, there is a Rokhlin invariant $R(\Sigma) \in \mathbb{Z}_{16}$ which is defined to be the residue class of the signature of any (smooth) spin 4-manifold with boundary $\Sigma$. It is easy to see that the Rokhlin invariant of a $\mathbb{Z}_{2}$-homology sphere is always even. The Rokhlin invariant defines a homomorphism

$$
R: \Theta_{\mathbb{Z}_{2}}^{3} \rightarrow \mathbb{Z}_{16}
$$

with image $2 \mathbb{Z}_{16}$ (the Rokhlin invariant of $L(3,1)$ is two).

Definition 1. Let $\Sigma$ be an oriented $\mathbb{Z}_{2}$-homology sphere. We define

$$
\begin{aligned}
& m(\Sigma)=\max \left\{\frac{5}{4} \sigma(X)-b_{2}(X) \mid w_{2}(X)=0, \partial X=\Sigma\right\} \\
& \bar{m}(\Sigma)=\min \left\{\frac{5}{4} \sigma(X)+b_{2}(X) \mid w_{2}(X)=0, \partial X=\Sigma\right\}
\end{aligned}
$$

Here $X$ runs over all smooth spin 4-manifolds with boundary $\Sigma$ and $\sigma(X)$ denotes the signature of $X$.

Surprisingly enough this simple definition actually yields bordism invariants which can be estimated if the manifold in question is a double covering of the 3 -sphere branched along some knot. The following theorem summarizes some properties of the invariants $m$ and $\bar{m}$. 


\section{Theorem 1.}

(1) The invariants $m$ and $\bar{m}$ are finite numbers. Moreover $m(\Sigma) \leq \bar{m}(\Sigma)$ where equality occurs if and only if $m(\Sigma)=\bar{m}(\Sigma)=0$ and $R(\Sigma)=0$.

(2) For every $\mathbb{Z}_{2}$-homology sphere $\Sigma, m(-\Sigma)=-\bar{m}(\Sigma)$.

(3) If $\Sigma_{1}$ and $\Sigma_{2}$ are $\mathbb{Z}_{2}$-homology spheres, then

$$
\begin{aligned}
& m\left(\Sigma_{1} \# \Sigma_{2}\right) \geq m\left(\Sigma_{1}\right)+m\left(\Sigma_{2}\right) \\
& \bar{m}\left(\Sigma_{1} \# \Sigma_{2}\right) \leq \bar{m}\left(\Sigma_{1}\right)+\bar{m}\left(\Sigma_{2}\right)
\end{aligned}
$$

(4) If $\Sigma_{1}$ and $\Sigma_{2}$ are $\mathbb{Z}_{2}$-homology cobordant then we have $m\left(\Sigma_{1}\right)=m\left(\Sigma_{2}\right)$ and $\bar{m}\left(\Sigma_{1}\right)=\bar{m}\left(\Sigma_{2}\right)$. In particular $m(\Sigma)=\bar{m}(\Sigma)=0$ if $\Sigma$ is the boundary of a $\mathbb{Z}_{2}$-acyclic 4 -manifold.

(5) If $m(\Sigma)>0$ or $\bar{m}(\Sigma)<0$ then $\Sigma$ has infinite order in the group $\Theta_{\mathbb{Z}_{2}}^{3}$. The same is true if $m(\Sigma)=0$ and $R(\Sigma) \neq 0$.

(6) If $\Sigma$ is a double covering of the 3-sphere branched along a knot $K$, then

$$
\frac{5}{4} \sigma(K)-2 g^{*}(K) \leq m(\Sigma) \leq \bar{m}(\Sigma) \leq \frac{5}{4} \sigma(K)+2 g^{*}(K)
$$

where $g^{*}(K)$ denotes the slice genus of $K$ and $\sigma(K)$ its signature.

Proof. For the proof of statement (1), let $\Sigma$ be a $\mathbb{Z}_{2}$-homology sphere. Pick a spin manifold $X$ with boundary $\Sigma$. Now suppose we are given another spin manifold $Y$ such that $\partial Y=\Sigma$. Let $W=Y \cup(-X)$. As $\Sigma$ is a $\mathbb{Z}_{2}$-homology sphere, $W$ is spin and $b_{2}(W)=b_{2}(X)+b_{2}(Y), \sigma(W)=\sigma(Y)-\sigma(X)$. Hence

$$
\begin{aligned}
\frac{5}{4} \sigma(Y)-b_{2}(Y) & =\frac{5}{4}(\sigma(Y)-\sigma(X)+\sigma(X))-b_{2}(Y) \\
& =\frac{5}{4}(\sigma(Y)-\sigma(X))+\frac{5}{4} \sigma(X)-b_{2}(Y) \\
& \leq b_{2}(W)-b_{2}(Y)+\frac{5}{4} \sigma(X)=b_{2}(X)+\frac{5}{4} \sigma(X)
\end{aligned}
$$

where we used Furuta's Theorem [13] in the third line. Since $b_{2}(X)+\frac{5}{4} \sigma(X)$ does not depend on $Y$, we can conclude that $m(\Sigma)$ is finite and bounded from above by $\frac{5}{4} \sigma(X)+b_{2}(X)$. As this is true for every $X$, we also obtain that $\bar{m}(\Sigma) \geq m(\Sigma)$ and the finiteness of $\bar{m}$. Now suppose we are given a $\mathbb{Z}_{2}$-homology sphere $\Sigma$ such that $m(\Sigma)=\bar{m}(\Sigma)$. Then we can find spin 4-manifolds $X$ and $Y$ with $\partial X=\partial Y=\Sigma$ such that

$$
\bar{m}(\Sigma)=\frac{5}{4} \sigma(X)+b_{2}(X)=\frac{5}{4} \sigma(Y)-b_{2}(Y)=m(\Sigma)
$$

Consider the spin manifold $W=-X \cup Y$. Since $\sigma(W)=\sigma(Y)-\sigma(X)$ and $b_{2}(W)=b_{2}(X)+b_{2}(Y)$, the above equality implies that $\frac{5}{4} \sigma(W)=b_{2}(W)$. By Furuta's Theorem, this is only possible if $b_{2}(W)=0$, and we obtain that $b_{2}(X)=$ $b_{2}(Y)=0$. Hence we have $m(\Sigma)=\bar{m}(\Sigma)=0$ and $R(\Sigma)=0$ as claimed. 
Now let us prove property (2). Pick a spin manifold $X$ with boundary $-\Sigma$ such that $\frac{5}{4} \sigma(X)+b_{2}(X)=\bar{m}(-\Sigma)$. Then $-X$ has boundary $\Sigma$, and we obtain that

$$
-\frac{5}{4} \sigma(X)-b_{2}(X)=-\bar{m}(-\Sigma) \leq m(\Sigma) .
$$

Hence we have $-m(\Sigma) \leq \bar{m}(-\Sigma)$. If we choose a spin manifold $Y$ with boundary $\Sigma$ such that $\frac{5}{4} \sigma(Y)-b_{2}(Y)=m(\Sigma)$, then the boundary of $-Y$ is $-\Sigma$, and we have

$$
-\frac{5}{4} \sigma(Y)+b_{2}(Y)=-m(\Sigma) \geq \bar{m}(-\Sigma) \text {. }
$$

These two inequalities imply the desired result.

As to assertion (3), first note that - thanks to property (2) - it suffices to prove this for $m$. Choose spin manifolds $X_{1}$ and $X_{2}$ with boundaries $\Sigma_{1}$ and $\Sigma_{2}$ such that $\frac{5}{4} \sigma\left(X_{i}\right)-b_{2}\left(X_{i}\right)=m\left(\Sigma_{i}\right)$. Then $V=X_{1} \natural X_{2}$ is spin with boundary $\Sigma_{1} \# \Sigma_{2}$ and $\frac{5}{4} \sigma(V)-b_{2}(V)=m\left(\Sigma_{1}\right)+m\left(\Sigma_{2}\right)$.

To prove assertion (4), note that again we only have to prove the required property for $m$. The condition $\left[\Sigma_{1}\right]=\left[\Sigma_{2}\right]$ implies that there exists a spin 4manifold $W$ with boundary $\partial W=\Sigma_{1}-\Sigma_{2}$ such that $\sigma(W)=b_{2}(W)=0$. Pick manifolds $X_{1}, X_{2}$ such that $\partial X_{i}=\Sigma_{i}$ and $\frac{5}{4} \sigma\left(X_{i}\right)-b_{2}\left(X_{i}\right)=m\left(\Sigma_{i}\right)$. Consider the spin manifolds

$$
\begin{aligned}
& V_{1}=X_{1} \cup_{\Sigma_{1}}(-W) \\
& V_{2}=X_{2} \cup_{\Sigma_{2}} W
\end{aligned}
$$

Then $\partial V_{1}=\Sigma_{2}, \sigma\left(V_{1}\right)=\sigma\left(X_{1}\right)$ and $b_{2}\left(V_{1}\right)=b_{2}\left(X_{1}\right)$. Similarly $\partial V_{2}=\Sigma_{1}$, $\sigma\left(V_{2}\right)=\sigma\left(X_{2}\right)$ and $b_{2}\left(V_{2}\right)=b_{2}\left(X_{2}\right)$. So we obtain

$$
\begin{aligned}
& m\left(\Sigma_{2}\right) \geq \frac{5}{4} \sigma\left(V_{1}\right)-b_{2}\left(V_{1}\right)=m\left(\Sigma_{1}\right) \\
& m\left(\Sigma_{1}\right) \geq \frac{5}{4} \sigma\left(V_{2}\right)-b_{2}\left(V_{2}\right)=m\left(\Sigma_{2}\right)
\end{aligned}
$$

and the claim follows. To prove the second part of the assertion, we only have to show that $m\left(S^{3}\right)=\bar{m}\left(S^{3}\right)=0$. Since $b_{2}\left(D^{4}\right)=\sigma\left(D_{4}\right)=0$, we have $m\left(S^{3}\right) \geq 0$ and $\bar{m}\left(S^{3}\right) \leq 0$. By statement $(1)$, we obtain

$$
0 \leq m\left(S^{3}\right) \leq \bar{m}\left(S^{3}\right) \leq 0
$$

and therefore $m\left(S^{3}\right)=\bar{m}\left(S^{3}\right)=0$.

The first part of statement (5) is an immediate consequence of (4) and (3). As to the second part, the assumption $m(\Sigma)=0$ implies that there exists a spin manifold $X$ with boundary $\Sigma$ such that $\frac{5}{4} \sigma(X)=b_{2}(X)$. Now suppose there is some $n>0$ such that $n \Sigma$ is the boundary of a $\mathbb{Z}_{2}$-acyclic manifold $V$. Consider the spin manifold

$$
Y=\underbrace{X \cup X \cdots X}_{n \text { copies }} \cup-V
$$

Then $\frac{5}{4} \sigma(Y)=b_{2}(Y)$ which, by Furuta's Theorem, implies that $\sigma(Y)=0$. But $\sigma(Y)=n \sigma(X)$, hence we obtain that $\sigma(X)=0$, in contradiction to $R(\Sigma) \neq 0$. 


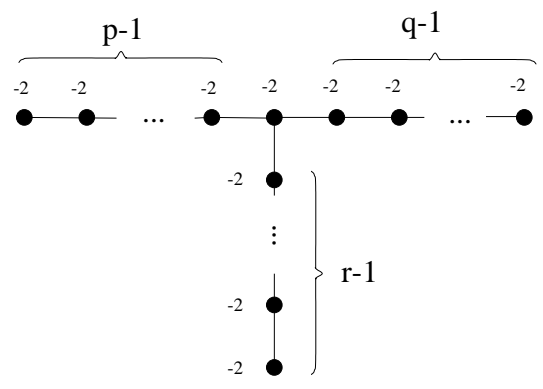

Figure 1. The graph $T_{p, q, r}$

To prove property 1 , note that given a surface $F$ in $D^{4}$ with boundary $K$, there is a double covering $X \rightarrow D^{4}$ branched along $F$ with boundary $\Sigma$. The manifold $X$ is spin, and it is well known that $b_{2}(X)=2 g(F)$ whereas $\sigma(X)=\sigma(K)$ (see [6] for a proof). This implies the claimed inequalities.

Remark 1. Observe that the invariants $m$ and $\bar{m}$ are both not additive. In fact, if one of them were additive, then property (2) would imply that $m(\Sigma)=\bar{m}(\Sigma)$ for all $\mathbb{Z}_{2}$-homology spheres $\Sigma$. As there are clearly $\mathbb{Z}_{2}$-homology spheres with non-zero Rokhlin invariant - for instance $\Sigma=L(3,1)$ - this is impossible by property (1).

We also note that the invariants $m$ and $\bar{m}$ are in general not integers (however they are always multiples of $\frac{1}{2}$ ) and that they are related by the formula

$$
m(\Sigma)=\bar{m}(\Sigma)=\frac{1}{4} R(\Sigma) \bmod 2 \mathbb{Z}
$$

to the Rokhlin invariant. In particular $m(\Sigma)-\bar{m}(\Sigma)$ is always an even integer.

Example 1. Suppose we are given positive integers $p \leq q \leq r$ such that exactly one of these three numbers is even, $\frac{1}{p}+\frac{1}{q}+\frac{1}{r}<1$ and $p+q+r \leq 22$. Let $T_{p, q, r}$ denote the weighted graph shown in figure 1 and consider the 4 -manifold $X_{p, q, r}$ obtained by plumbing according to $T_{p, q, r}$. It is not hard to check that the determinant of $T_{p, q, r}$ is - up to sign - the number $p q r-p q-p r-q r$ and is therefore odd, hence the boundary $\Sigma_{p, q, r}=\partial X_{p, q, r}$ is a $\mathbb{Z}_{2}$-homology sphere.

As the signature of $T_{p, q, r}$ is $4-p-q-r$ and the rank is $p+q+r-2$, we obtain that

$$
\bar{m}\left(\Sigma_{p, q, r}\right) \leq-\frac{1}{4}(p+q+r)+3 .
$$

Now it has been proved in [18] and [20] that the bilinear form $T_{p, q, r}$ can be realized by a collection of $(-2)$-curves in a K3-surface, and a regular neighborhood of such a configuration of spheres is diffeomorphic to $X_{p, q, r}$, hence we have an embedding $X_{p, q, r} \subset K 3$. Let $Y$ denote the closure of the complement. Then $\partial Y=-\Sigma_{p, q, r}$, $\operatorname{sign}(Y)=-16-\operatorname{sign}\left(X_{p, q, r}\right)=p+q+r-20$ and $b_{2}(Y)=22-b_{2}\left(X_{p, q, r}\right)=$ 
$24-p-q-r$, and we obtain

$$
m\left(\Sigma_{p, q, r}\right) \geq-\frac{5}{4}(p+q+r-20)-24+p+q+r=-\frac{1}{4}(p+q+r)+1 .
$$

Now we claim that $\bar{m}\left(\Sigma_{p, q, r}\right)>m\left(\Sigma_{p, q, r}\right)$. In fact, if $p+q+r \not \equiv 4 \bmod 16$, this follows from Theorem 1 , as in this case $R\left(\Sigma_{p, q, r}\right) \neq 0$. Our conditions on $p, q, r$ exclude the case that $p+q+r=4$, so the only remaining case we have to check is $p+q+r=20$. But then we already know that $\bar{m}\left(\Sigma_{p, q, r}\right) \leq-2$, and therefore we can again conclude that $\bar{m}\left(\Sigma_{p, q, r}\right)>m\left(\Sigma_{p, q, r}\right)$, which proves our claim.

As the difference $m-\bar{m}$ is always an even integer, this discussion shows that the above estimates for $m\left(\Sigma_{p, q, r}\right)$ and $\bar{m}\left(\Sigma_{p, q, r}\right)$ are sharp, i.e.

$$
m\left(\Sigma_{p, q, r}\right)=-\frac{1}{4}(p+q+r)+1=\bar{m}\left(\Sigma_{p, q, r}\right)-2 .
$$

Note that $\Sigma_{2,3,7}$ is minus the Brieskorn sphere $\Sigma(2,3,7)$, hence we can conclude that $m(\Sigma(2,3,7))=0$. As $R(\Sigma(2,3,7))=8 \neq 0$, we obtain that $\Sigma(2,3,7)$ has infinite order in the group $\Theta_{\mathbb{Z}_{2}}^{3}$ although it bounds a rational ball [9]. This also follows from the results in [23].

As we have seen in Example 1, the Brieskorn sphere $\Sigma(2,3,7)$ is an element of infinite order in the kernel of the natural map $\Theta_{\mathbb{Z}_{2}}^{3} \rightarrow \Theta_{\mathbb{Q}}^{3}$, where the latter group is defined in the obvious way. Also note that this map is not onto, as the Rokhlin invariant of every $\mathbb{Z}_{2}$-homology sphere is even. A similar argument shows that $\Theta_{\mathbb{Z}}^{3} \rightarrow \Theta_{\mathbb{Z}_{2}}^{3}$ is not onto. It is conceivable that this map is also not one-to-one, and it would be interesting to find an explicit example of an integral homology sphere which is not the boundary of a $\mathbb{Z}$-acyclic 4 -manifold but the boundary of some $\mathbb{Z}_{2}$-acyclic manifold. We remark that Furuta's arguments in [12] actually show that the family $\Sigma(2 k+1,4 k+1,4 k+3)$ of Brieskorn spheres generates a subgroup of infinite order in $\Theta_{\mathbb{Z}_{2}}^{3}$ (this has been used by H. Endo [7] to prove that the corresponding family of Pretzel knots spans an infinite dimensional subgroup in the smooth knot concordance group) and that the restriction of $\Theta_{\mathbb{Z}}^{3} \rightarrow \Theta_{\mathbb{Z}_{2}}^{3}$ to this subgroup is one-to-one.

Corollary 1. Suppose we are given positive integers $p \leq q \leq r$ such that exactly one of these three numbers is even, $\frac{1}{p}+\frac{1}{q}+\frac{1}{r}<1$ and $p+q+r \leq 22$. Then the Montesinos knot $\mathfrak{m}(2 ;(p, p-1),(q, q-1),(r, r-1))$ has slice genus and unknotting number $\frac{1}{2}(p+q+r)-1$.

Proof. Let $K$ denote the knot $\mathfrak{m}(2 ;(p, p-1),(q, q-1),(r, r-1))$. The double covering $\Sigma$ of $S^{3}$ branched along this knot is $-\Sigma_{p, q, r}$, hence

$$
m(\Sigma)=-\bar{m}\left(\Sigma_{p, q, r}\right)=\frac{1}{4}(p+q+r)-3
$$

by Example 1. It is not hard to verify that $\sigma(K)=p+q+r-4$. By Theorem 1 
we obtain that

$$
2 g^{*}(K) \geq \frac{5}{4} \sigma(K)-m(\Sigma)=\frac{5}{4}(p+q+r)-5-\frac{1}{4}(p+q+r)+3=p+q+r-2,
$$

so $g^{*}(K) \geq \frac{1}{2}(p+q+r)-1$. Now the knot $K$ is build up from the rational tangles $t(p, p-1), t(q, q-1)$ and $t(r, r-1)$. The rational tangle $t(p, p-1)$ can be changed to the trivial tangle by $\frac{p}{2}$ crossing changes if $p$ is even and by $\frac{p-1}{2}$ crossing changes if $p$ is odd, so we obtain that $u(K) \leq \frac{1}{2}(p+q+r)-1$. Of course $g^{*}(K) \leq u(K)$, and therefore we can conclude that $u(K)=g^{*}(K)=\frac{1}{2}(p+q+r)-1$.

Note that the Montesinos knots in Corollary 1 are examples of knots where the inequality $g^{*}(K) \geq \frac{1}{2}|\sigma(K)|$ from [19] is not sharp. In some sense the last statement of Theorem 1 can be seen as a refinement of this inequality involving the cobordism class of the double branched covering.

Example 2. Suppose that $\Sigma$ is an integral homology sphere with non-zero Rokhlin invariant which is the result of rational surgery on a torus knot. Then $m(\Sigma) \geq 0$. This follows from [23], where Saveliev shows that certain Brieskorn spheres bound spin 4-manifolds $X$ with intersection forms $-a E_{8}+b H, a \geq b$ and uses this to prove that they have infinite order in the homology cobordism group. For these homology spheres $m(\Sigma) \geq \frac{5}{4} \sigma(X)-b_{2}(X)=2(a-b) \geq 0$.

\section{Some computations for lens spaces}

As A. Casson and J. Harer demonstrated in [5], all lens spaces of the form $L\left(t^{2}, q t+1\right)$ with coprime numbers $t$ and $q$ where $t$ is odd bound $\mathbb{Z}_{2}$-acyclic 4manifolds, hence they are zero in the group $\Theta_{\mathbb{Z}_{2}}^{3}$. In this section, we prove estimates for the invariants $m$ and $\bar{m}$ of lens spaces and use them to find many examples of lens spaces which have in fact infinite order in the $\mathbb{Z}_{2}$-homology cobordism group. In particular we will obtain a complete list of those lens spaces $L(\alpha, \beta)$ with odd $\alpha \leq 11$ whose cobordism classes have infinite order.

It is well known that a lens space $L(\alpha, \beta)$ is a double covering of the 3 -sphere branched along the two-bridge link $S(\alpha, \beta)$, see for instance [4]. To be able to apply statement 1 in Theorem 1 , we have to compute respectively to estimate the signature and the slice genus of such a link. Let us start by fixing some notations.

Definition 2. Assume that we are given a sequence $\left(a_{1}, b_{1}, a_{2}, \cdots, a_{n}\right)$ of $2 n-$ 1 non-zero integers. Then we define the link $P\left(a_{1}, b_{1}, \cdots, a_{n}\right)$ to be the 4-plat obtained by closing the 3 -string braid $\sigma_{2}^{-a_{1}} \sigma_{1}^{2 b_{1}} \cdots \sigma_{2}^{-a_{n}}$ as pictured in figure 2 (here we use the convention that the generators of the braid group have positive crossings if the two strings involved have parallel orientations).

The link $P\left(a_{1}, b_{1}, \cdots, a_{n}\right)$ is a knot if and only if $\sum_{i} a_{i}$ is odd, otherwise it is 


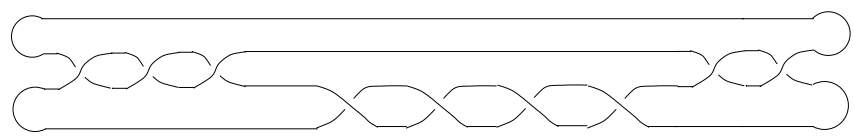

Figure 2. $\mathrm{P}(3,2,2)$

a two component link.

Definition 3. Assume that we are given coprime integers $0<\beta<\alpha$ such that $\beta$ is odd. Then an admissible continuous fractions decomposition is a presentation of $\frac{\alpha}{\beta}$ as a continuous fraction

$$
\frac{\alpha}{\beta}=\left[a_{1}, 2 b_{1}, a_{2}, \cdots a_{n}\right]=a_{1}+\frac{1}{2 b_{1}+\frac{1}{a_{2}+\frac{1}{2 b_{2}+\cdots}}}
$$

where the $a_{i}$ and $b_{i}$ are integers such that $a_{i} b_{i}>0$ for all $i=1, \cdots, n-1$.

Observe that such an admissible continuous fractions decomposition exists for every pair $0<\beta<\alpha$ with $\operatorname{gcd}(\alpha, \beta)=1$ and $\beta \equiv 1 \bmod 2$. It is also well known [4] that the 4-plat $P\left(a_{1}, b_{1}, \cdots, a_{n}\right)$ is nothing else than the 2-bridge link $S(\alpha, \beta)$. This description of 2-bridge links turns out to be particularly useful for computing the signature and slice genus of such a link. The following fact can be found in [3].

Lemma 1 (see [3]]). Assume that $0<\beta<\alpha$ are coprime integers and that $\beta$ is odd. Pick an admissible continuous fractions expansion

$$
\frac{\alpha}{\beta}=\left[a_{1}, 2 b_{1}, \cdots, a_{n}\right] .
$$

Then the signature of the two-bridge link $S(\alpha, \beta)$ is given by

$$
\sigma(S(\alpha, \beta))=\sum_{i} a_{i}-\frac{a_{n}}{\left|a_{n}\right|} .
$$

To find an upper bound for the slice genus of a two-bridge knot we will use the following general observation.

Proposition 1. Suppose that $K_{1}$ and $K_{2}$ are links such that $K_{2}$ is obtained from $K_{1}$ by $p$ positive and $n$ negative crossing changes. Then

$$
g^{*}\left(K_{1}\right) \leq g^{*}\left(K_{2}\right)+\max \{p, n\} .
$$

Proof. The trace of a homotopy given by the crossing changes is a union of immersed annuli $A$ in $S^{3} \times I$ with $p$ positive and $n$ negative self-intersection points 
connecting $K_{1} \subset S^{3} \times\{0\}$ and $K_{2} \subset S^{3} \times\{1\}$ (see [6]). Pick a connected surface $F \subset D^{4}$ with boundary $K_{2}$ such that $g(F)=g^{*}\left(K_{2}\right)$. By gluing this surface with $A$ we obtain a connected immersed surface $F^{\prime} \subset D^{4}=\left(S^{3} \times I\right) \cup D^{4}$ having boundary $K_{1}$ whose genus is $g(F)$ and which has $p$ positive and $n$ negative self intersection points. Now let us assume that $p \geq n$. Since we can join two self-intersection points of opposite signs by a handle we can construct a surface bounding $K_{1}$ which has genus $g(F)+n$ and $p-n$ positive self-intersection points. Replacing the remaining self-intersections points by $p-n$ handles, we end up with an embedded surface with boundary $K_{1}$ which has genus $g(F)+p$ and the claim follows. In the case that $n \geq p$ a similar argument applies.

Lemma 2. Suppose that we have a sequence $\left(a_{1}, b_{1}, a_{2}, \cdots, a_{n}\right)$ of non-zero integers such that $\sum_{i} a_{i}$ is odd. Define numbers $o^{+}, o^{-}$by

$$
\begin{aligned}
& o^{+}=\#\left\{1 \leq i \leq n \mid a_{i} \equiv 1 \bmod 2, a_{i}>0\right\}, \\
& o^{-}=\#\left\{1 \leq i \leq n \mid a_{i} \equiv 1 \bmod 2, a_{i}<0\right\} .
\end{aligned}
$$

Then the slice genus $g^{*}$ of the knot $P\left(a_{1}, b_{1}, \cdots, a_{n}\right)$ is bounded from above by

$$
g^{*} \leq \frac{1}{4} \max \left\{\sum_{i}\left(\left|a_{i}\right|-a_{i}\right)+2 o^{+}-2, \sum_{i}\left(\left|a_{i}\right|+a_{i}\right)+2 o^{-}-2\right\} .
$$

Proof. Suppose that we have an index $0 \leq i \leq n$ such that $a_{i}>0$. If $a_{i}$ is odd, we can deform the knot $P\left(a_{1}, b_{1}, \cdots, a_{n}\right)$ into the knot defined by the sequence $\left(a_{1}, b_{1}, \cdots, b_{i-1}, 1, b_{i}, a_{i+1}, \cdots, a_{n}\right)$ by performing $\frac{1}{2}\left(a_{i}-1\right)$ negative crossing changes. If $a_{i}>0$ is even, we can do $\frac{1}{2} a_{i}$ negative crossing changes to obtain $P\left(a_{1}, b_{1}, \cdots, b_{i-1}, 0, b_{i}, a_{i+1}, \cdots, a_{n}\right)$. Repeating this for every index $i$ for which $a_{i}$ is positive, we eventually obtain a knot $P\left(a_{1}^{\prime}, b_{1}, a_{2}^{\prime}, b_{2}, \cdots, a_{n}\right)$ for which $a_{i}^{\prime}=a_{i}$ if $a_{i}<0$ and $a_{i}^{\prime} \in\{0,1\}$ otherwise after having performed

$$
n=\sum_{\substack{i \\ a_{i}>0 \\ a_{i} \text { odd }}} \frac{1}{2}\left(a_{i}-1\right)+\sum_{\substack{i \\ a_{i}>0 \\ a_{i} \text { even }}} \frac{1}{2} a_{i}=\frac{1}{4}\left(\sum_{i}\left(\left|a_{i}\right|+a_{i}\right)-2 o^{+}\right)
$$

negative crossing changes.

A similar reduction can be done if $a_{i}<0$. In this case we can do $\frac{1}{2}\left(\left|a_{i}\right|-1\right)$ respectively $\frac{1}{2}\left|a_{i}\right|$ positive crossing changes, depending on whether $a_{i}$ is odd or even. So we see that after performing

$$
p=\frac{1}{4}\left(\sum_{i}\left(\left|a_{i}\right|-a_{i}\right)-2 o^{-}\right)
$$

additional positive crossing changes, we end up with a link $P\left(a_{1}^{\prime \prime}, b_{1}, a_{2}^{\prime \prime}, b_{2}, a_{n}^{\prime \prime}\right)$ where $a_{i}^{\prime \prime} \in\{-1,0,1\}$ and $a_{i}^{\prime \prime}=0$ if and only if $a_{i}$ is even.

Observe that the knot $P\left(a_{1}^{\prime \prime}, b_{1}, a_{2}^{\prime \prime}, b_{2}, a_{n}^{\prime \prime}\right)$ is the boundary of an obvious Seifert surface which has genus $\frac{1}{2}\left(o^{+}+o^{-}-1\right)$. 
Now assume that $p \geq n$, i.e. $p=\max \{p, n\}$. By Proposition 1 we can conclude that

$$
\begin{aligned}
g^{*}(S(\alpha, \beta)) \leq p+g(F) & =\frac{1}{4}\left(\sum_{i}\left(\left|a_{i}\right|-a_{i}\right)-2 o^{-}\right)+\frac{1}{2}\left(o^{+}+o^{-}-1\right) \\
& =\frac{1}{4}\left(\sum_{i}\left(\left|a_{i}\right|-a_{i}\right)+2 o^{+}-2\right)
\end{aligned}
$$

If we have $n \geq p$, we can use the same argument to obtain the lower bound

$$
\begin{aligned}
g^{*}(S(\alpha, \beta)) \leq n & +g(F)=\frac{1}{4}\left(\sum_{i}\left(\left|a_{i}\right|+a_{i}\right)-2 o^{+}\right)+\frac{1}{2}\left(o^{+}+o^{-}-1\right) \\
& =\frac{1}{4}\left(\sum_{i}\left(\left|a_{i}\right|+a_{i}\right)+2 o^{-}-2\right) .
\end{aligned}
$$

Since of course either $p \geq n$ or $n \geq p$ the claimed inequality follows.

In the case that all the $a_{i}$ are positive, it follows from work of T. Tanaka [24] and L. Rudolph [21] that this estimate is actually sharp.

Proposition 2. Suppose that $0<\beta<\alpha$ are coprime odd numbers and that we are given a continuous fractions decomposition

$$
\frac{\alpha}{\beta}=\left[a_{1}, 2 b_{1}, a_{2}, \cdots a_{n}\right]
$$

with non-zero integers $a_{i}, b_{i}$ such that $a_{i} b_{i}>0$ for all $i$. Let

$$
\begin{aligned}
& o^{+}=\#\left\{1 \leq i \leq n \mid a_{i} \equiv 1 \bmod 2, a_{i}>0\right\}, \\
& o^{-}=\#\left\{1 \leq i \leq n \mid a_{i} \equiv 1 \bmod 2, a_{i}<0\right\} .
\end{aligned}
$$

Then

$$
\begin{aligned}
m(L(\alpha, \beta)) \geq \frac{5}{4}\left(\sum_{i=1}^{n} a_{i}-\frac{a_{n}}{\left|a_{n}\right|}\right)-\frac{1}{2} \max \{ & \sum_{i=1}^{n}\left(\left|a_{i}\right|+a_{i}\right)+2 o^{-}-2, \\
& \left.\sum_{i=1}^{n}\left(\left|a_{i}\right|-a_{i}\right)+2 o^{+}-2\right\} .
\end{aligned}
$$

and

$$
\begin{aligned}
\bar{m}(L(\alpha, \beta)) \leq \frac{5}{4}\left(\sum_{i=1}^{n} a_{i}-\frac{a_{n}}{\left|a_{n}\right|}\right)+\frac{1}{2} \max \{ & \sum_{i=1}^{n}\left(\left|a_{i}\right|+a_{i}\right)+2 o^{-}-2, \\
& \left.\sum_{i=1}^{n}\left(\left|a_{i}\right|-a_{i}\right)+2 o^{+}-2\right\} .
\end{aligned}
$$

Proof. It is well known that the lens space $L(\alpha, \beta)$ is a double covering of $S^{3}$, branched along the two-bridge knot $S(\alpha, \beta)=P\left(a_{1}, b_{1}, \cdots, a_{n}\right)$, see for instance 


\begin{tabular}{|r|r|r|r|r|}
\hline$L(\alpha, \beta)$ & $m \geq$ & $\bar{m} \leq$ & {$\left[a_{1}, 2 b_{2}, a_{2}, \cdots, a_{n}\right]$} & order \\
\hline$(3,1)$ & 0.5 & 4.5 & {$[3]$} & $\infty$ \\
$(5,3)$ & -2.0 & 2.0 & {$[1,2,-2]$} & $\leq 2$ \\
$(7,1)$ & 1.5 & 13.5 & {$[7]$} & $\infty$ \\
$(7,3)$ & 0.5 & 4.5 & {$[2,4,-1]$} & $\infty$ \\
$(9,1)$ & 2.0 & 18.0 & {$[9]$} & $\infty$ \\
$(9,5)$ & -2.0 & 2.0 & {$[1,2,-1,-2,-1]$} & 0 \\
$(11,1)$ & 2.5 & 22.5 & {$[11]$} & $\infty$ \\
$(11,3)$ & 0.5 & 4.5 & {$[3,2,-2]$} & $\infty$ \\
$(11,5)$ & 0.5 & 4.5 & {$[2,6,-1]$} & $\infty$ \\
$(13,1)$ & 3.0 & 27.0 & {$[13]$} & $\infty$ \\
$(13,3)$ & 1.0 & 9.0 & {$[4,4,1]$} & $\infty$ \\
$(13,5)$ & -2.0 & 2.0 & {$[2,2,-3]$} & $\leq 2$ \\
$(13,7)$ & -2.0 & 2.0 & {$[1,2,-1,-4,-1]$} & $?$ \\
\hline
\end{tabular}

Table 1. Bounds for $m(L(p, q))$

[4]. By Lemma 2, there exists a surface $F \subset D^{4}$ with boundary $S(\alpha, \beta)$ which has genus

$$
g(F)=\frac{1}{4} \max \left\{\sum_{i}\left(\left|a_{i}\right|-a_{i}\right)+2 o^{+}-2, \sum_{i}\left(\left|a_{i}\right|+a_{i}+2 o^{-}-2\right)\right\} .
$$

Using the expression for the signature of $S(\alpha, \beta)$ derived in Lemma 1, Theorem 1 now immediately yields the desired result.

Remark 2. As to the orientation of lens spaces, we are using the convention from [4] that the oriented lens space $L(\alpha, \beta)$ for $0<\beta<\alpha$ is the double covering of the 3 -sphere branched along the two-bridge link $S(\alpha, \beta)$. With this choice of orientations, the covering of the left-handed trefoil knot is $L(3,1)$. Note that this is in accordance with the convention used in [14], where the lens space $L(\alpha, \beta)$ is defined to be the result of rational surgery along the unknot with framing $\frac{-\alpha}{\beta}$ (see [14], Exercise 6.3.5).

By computing the terms appearing in the statement of Proposition 2 we can find many examples of lens spaces which have infinite order in the $\mathbb{Z}_{2}$-homology cobordism group. Table 1 shows - up to orientation - all the lens spaces whose first homology groups have odd order less or equal than 13 together with the estimates for $m$ and $\bar{m}$ provided by Proposition 2, the continuous fractions decomposition used for the computation and the order of the lens space in $\Theta_{\mathbb{Z}_{2}}^{3}$ as far as it is known (note that the lens spaces $L(5,3)$ and $L(13,5)$ have orientation reversing diffeomorphisms, the fact that $L(9,5)$ is the boundary of a $\mathbb{Z}_{2}$-acyclic manifold is proved in [5])). 
Corollary 2. Suppose that $0<\beta<\alpha$ are coprime odd numbers and that $\frac{\alpha}{\beta}$ has a continuous fractions decomposition

$$
\frac{\alpha}{\beta}=\left[a_{1}, 2 b_{1}, a_{2}, 2 b_{2}, a_{3}, \cdots, a_{n}\right]
$$

such that $a_{i}, b_{i}>0$ for all $i$. Then the lens space $L(\alpha, \beta)$ has infinite order in the $\mathbb{Z}_{2}$-homology cobordism group.

Example 3. Suppose $n>0$ is some positive integer. Then the lens space $L(10 n+1,8 n+1)$ has infinite order in $\Theta_{\mathbb{Z}_{2}}^{3}$. In fact, first note that $10 n+1$ and $8 n+1$ are odd and coprime (if some prime $p$ divides $10 n+1$ and $8 n+1$, then $2 a \equiv 0 \bmod p$, in contradiction to $10 n+1 \equiv 0 \bmod p)$. A continuous fractions decomposition is given by

$$
\frac{10 n+1}{8 n+1}=1+\frac{2 n}{8 n+1}=1+\frac{1}{4+\frac{1}{2 n}}=[1,4,2 n] .
$$

Since all the coefficients are positive Corollary 2 applies and the claim follows.

As the lens spaces $L(10 n+1,8 n+1)$ and $L(10 m+1,8 m+1)$ are diffeomorphic if and only if $n=m$, we obtain in particular that there is an infinite family of lens spaces which have infinite order in the $\mathbb{Z}_{2}$-homology cobordism group.

Remark 3. As the referee pointed out, one can also use the orbifold version of Furuta's Theorem [11] and arguments similar to those in [22] to prove Corollary 2 as well as the claims made in the last column of Table 1.

Example 4. We have seen that a $\mathbb{Z}_{2}$-homology sphere obtained by integral surgery on the unknot - namely a lens space $L(n, 1)$ - has infinite order in the $\mathbb{Z}_{2}$-homology cobordism group unless it is an integral homology sphere, in which case it is $\mathbb{Z}_{2^{-}}$ homology cobordant to $S^{3}$. It turns out that the same is true if the knot is slice, unless the framing is \pm 1 . In fact, suppose that $\Sigma$ is obtained by doing surgery with odd framing $n$ on a slice knot $K$. Without loss of generality we can assume that $n>0$. Let $W$ be the trace of the surgery, i.e. $W$ is obtained from $D^{4}$ by attaching a 2-handle along $K$ with framing $n$. Then an embedded disk in the 4-ball with boundary $K$ and the core of the 2-handle can be glued together to given an embedded sphere $S \subset W$ with self-intersection number $n$ which generates $H_{2}(W ; \mathbb{Z})$. Let $X$ be the manifold obtained from $W$ by removing a tubular neighborhood of $S$. As the boundary of such a neighborhood is a lens space $L(n, 1)$, we have $\partial X=\Sigma \cup L(n, 1)$. Now it is easy to see that $X$ is $\mathbb{Z}_{2}$-acyclic, hence $\Sigma$ is $\mathbb{Z}_{2}$-homology cobordant to $-L(n, 1)$. This implies that $\Sigma$ has infinite order in $\Theta_{\mathbb{Z}_{2}}^{3}$ if $n \neq \pm 1$. Note that in the case that $n= \pm 1, X$ is even a $\mathbb{Z}$-acyclic manifold and as $L(1,1)=S^{3}$ we obtain that the integral homology sphere $\Sigma$ is the boundary of a $\mathbb{Z}$-acyclic manifold.

We close this section with the remark that the above computations for lens 
spaces can be generalized to prove bounds for $m(\Sigma)$ and $\bar{m}(\Sigma)$ if $\Sigma$ is a Seifert fibred $\mathbb{Z}_{2}$-homology sphere, note that these spaces are double coverings of the 3 sphere branched along a Montesinos link. As the arguments and calculations are very similar to the computations in the (special) case of lens spaces we only state the result for one class of examples.

Example 5. Let $n$ be a positive integer and consider the Seifert fibred space $\Sigma_{n}=M((12,5),(7,3),(6 n+1,4 n+1) ;-7)$, which is the double coverings of $S^{3}$ branched along the Montesinos knot $K=\mathfrak{m}((12,5),(7,3),(6 n+1,4 n+1) ;-7)$. The obvious Seifert surface for this knot is obtained from Seifert surfaces for the two-bridge knots $S(12,5), S(7,3)$ and $S(6 n+1,4 n+1)$ by adding three bands, one of them having 7 half twists. Using this and the results about the signature and the slice genus of a two-bridge knot which we obtained in this section one easily derives that $\sigma(K)=-2(n+3)$ and $g^{*}(K) \leq n+3$. Note that, as for every knot, we have the inequality $2 g^{*}(K) \geq|\sigma(K)|$, so our estimate for the slice genus happens to be sharp.

Now we can conclude that $\bar{m}\left(\Sigma_{n}\right) \leq-\frac{1}{2}(n+3)$. By Theorem 1 this shows that $\Sigma_{n}$ has infinite order in $\Theta_{\mathbb{Z}_{2}}^{3}$. Also note that the order of the first homology of $\Sigma_{n}$ is linear in $n$, so $\Sigma_{n} \neq \Sigma_{m}$ if $m \neq n$, and we obtain an infinite family of Seifert fibred spaces which have infinite order in $\Theta_{\mathbb{Z}_{2}}^{3}$.

\section{A surgery formula}

As indicated in the introduction, a second main source of $\mathbb{Z}_{2}$-homology spheres is surgery on knots with odd integral framings. In this section, we shall see how one can obtain information on the invariants $m(\Sigma)$ and $\bar{m}(\Sigma)$ if a $\mathbb{Z}_{2}$-homology sphere $\Sigma$ is described by integral surgery on a knot. Combined with other methods for calculating these invariants, this provides a lower bound for the slice genus of such a knot. We also give examples of 3-manifolds which cannot be obtained by integral surgery on a knot.

Suppose we are given a knot $K$ in the 3 -sphere. Then surgery with odd framing on this knot yields a $\mathbb{Z}_{2}$-homology sphere $\Sigma$. First let us derive a relation between the Arf invariant of the knot, the framing and the Rokhlin invariant $R(\Sigma)$ of $\Sigma$. For this purpose recall that if $W$ is a simply connected 4-manifold whose boundary is a $\mathbb{Z}_{2}$-homology sphere and $F \subset W$ is a closed embedded characteristic surface, we have a well defined $\operatorname{Arf}$ invariant $\operatorname{Arf}(F) \in \mathbb{Z}_{2}$ which does only depend of the homology class of the surface and the Rokhlin invariant of the boundary (as one can see by gluing with a simply connected spin manifold with boundary $-\partial W$, note that the condition that $\partial W$ is a $\mathbb{Z}_{2}$-homology sphere implies that $F$ is still characteristic in the resulting closed 4-manifold).

Proposition 3. Let $K$ be a knot and assume that $n$ is some odd number. Let $\Sigma$ 
denote the $\mathbb{Z}_{2}$-homology sphere which is the result of surgery along $K$ with framing $n$. Then $n-\frac{n}{|n|} \equiv-R(\Sigma) \bmod 8$ and

$$
\operatorname{Arf}(K)=\frac{1}{8}\left(n-\frac{n}{|n|}+R(\Sigma)\right) \bmod 2 .
$$

Proof. Let $W$ denote the simply connected 4-manifold which is obtained from $D^{4}$ by adding a handle along $K$ with framing $n$. Then $\partial W=\Sigma$ is a $\mathbb{Z}_{2}$-homology sphere, in fact $\# H_{1}(\Sigma ; \mathbb{Z})=|n|$. Furthermore $b_{2}(W)=1$. As the boundary of $W$ is a $\mathbb{Z}_{2}$-homology sphere, the $\mathbb{Z}_{2}$-intersection form is non-degenerate and hence the fact that $b_{2}(W)=1$ implies that $W$ is not spin, i.e. $w_{2}(W)$ is the non-zero element of $H^{2}\left(W ; \mathbb{Z}_{2}\right)=\mathbb{Z}_{2}$. Pick a Seifert surface $F^{\prime}$ for $K$ and let $F \subset W$ denote the surface which is obtained by gluing $F^{\prime}$ with the core of the 2 -handle. Then $F \cdot F=n$, and therefore $\operatorname{sign}(W)=\frac{n}{|n|}$. Let $[F] \in H_{2}\left(W ; \mathbb{Z}_{2}\right)$ denote the homology class of $F$ and denote the Poincaré duality map $H_{2}\left(W ; \mathbb{Z}_{2}\right) \rightarrow H^{2}\left(W, \partial W ; \mathbb{Z}_{2}\right)$ by $P D_{W}$. Then the fact that the self-intersection of $F$ is odd implies that the image of $P D_{W}([F])$ under the restriction $\iota^{*}: H^{2}\left(W, \partial W ; \mathbb{Z}_{2}\right) \rightarrow H^{2}\left(W ; \mathbb{Z}_{2}\right)$ is the non-zero element, i.e. we have

$$
\iota^{*} P D_{W}([F])=w_{2}(W) .
$$

Now pick a spin manifold $V$ with boundary $-\Sigma$ and consider the closed manifold $X=W \cup V$. The fact that $\Sigma$ is a $\mathbb{Z}_{2}$-homology sphere implies that $H^{2}\left(X ; \mathbb{Z}_{2}\right)=$ $H^{2}\left(W ; \mathbb{Z}_{2}\right) \oplus H^{2}\left(V ; \mathbb{Z}_{2}\right)$. As $V$ is spin the surface $F$ represents $w_{2}(X)$, i.e. $F \subset X$ is characteristic. Therefore we can conclude that $F \cdot F \equiv \operatorname{sign}(X) \bmod 8$. By Novikov additivity, $\operatorname{sign}(X)=\operatorname{sign}(W)+\operatorname{sign}(V)=\frac{n}{|n|}+\operatorname{sign}(V)$. By definition of the Rokhlin invariant we also have that $\operatorname{sign}(V) \equiv-R(\Sigma) \bmod 8$, and therefore we obtain that $n \equiv \frac{n}{|n|}-R(\Sigma) \bmod 8$. By [10], we obtain

$$
\operatorname{Arf}(F)=\frac{1}{8}(F \cdot F-\operatorname{sign}(X))=\frac{1}{8}\left(n-\frac{n}{|n|}+R(\Sigma)\right) \bmod 2
$$

for the Arf invariant of the surface $F$. However it is also known [17] that $\operatorname{Arf}(F)=$ $\operatorname{Arf}(K)$, and the proof of the proposition is complete.

Corollary 3. Assume that a $\mathbb{Z}_{2}$-homology sphere $\Sigma$ is obtained by integral surgery on a knot. Then

$$
\# H_{1}(\Sigma ; \mathbb{Z})-1 \equiv \pm R(\Sigma) \bmod 8
$$

Here the sign is -1 if the framing is positive, otherwise it is +1 .

Proof. Suppose that $\Sigma$ is obtained by integral surgery on a knot $K$ with framing $n$. First let us consider the case that $n$ is positive (note that $n$ must be odd). Then $\# H_{1}(\Sigma ; \mathbb{Z})=n$, and by Proposition 3 , we have that $\# H_{1}(\Sigma ; \mathbb{Z})-1 \equiv$ $-R(\Sigma) \bmod 8$. If $n$ is negative, we obtain $-\# H_{1}(\Sigma ; \mathbb{Z})+1 \equiv-R(\Sigma) \bmod 8$, and multiplying this by minus one gives the desired result. 
Remark 4. If a lens space $L(p, q)$ can be obtained by integral surgery on a knot, then $q$ or $-q$ is a square modulo $\mathrm{p}$ by Proposition 1 in [8]. It is interesting that if $p$ is an odd prime, this criterion is actually equivalent to the congruence of Corollary 3, which can therefore be seen as a generalization of the result for lens spaces in [8]. In fact, assume that $q$ is a square $\bmod p$ (we can restrict ourselves to this case after possibly reversing the orientation), i.e. $\left(\begin{array}{l}q \\ p\end{array}\right)=1$. By [15], p. 137, we have

$$
\left(\begin{array}{l}
q \\
p
\end{array}\right)+6 p s(q, p) \equiv \frac{p+1}{2} \bmod 4
$$

where $s(q, p)$ is a Dedekind sum. It is also known [25] that $R(L(p, q)) \equiv \pm 4 p^{2} s(q, p)$ mod 8 (the sign depending on orientation conventions), note that, as $p$ is odd, the Rokhlin invariant is even and therefore $2 p^{2} s(q, p)$ is an integer. Multiplying equation (1) by $2 \mathrm{p}$ we therefore obtain

$$
12 p^{2} s(q, p) \equiv-4 p^{2} s(q, p) \equiv p(p-1) \bmod 8 .
$$

But $p$ is an odd integer, hence $p^{2} \equiv 1 \bmod 8$, and we end up with

$$
\pm R(L(p, q)) \equiv 4 p^{2} s(q, p) \equiv p-1=\# H_{1}(L(p, q) ; \mathbb{Z})-1 \bmod 8
$$

which is the prediction made by Corollary 3. A similar calculation shows that if the congruence of Corollary 3 holds for a lens space $L(p, q)$, one of the Jacobi symbols $\left(\begin{array}{c}q \\ p\end{array}\right)$ and $\left(\begin{array}{c}-q \\ p\end{array}\right)$ must be one, hence, as $p$ is a prime, $q$ or $-q$ is a square modulo $p$.

In some cases Corollary 3 can be used to show that certain 3-manifolds are not the result of integral surgery on a knot (although they can of course be obtained by integral surgery on a link) or to determine the sign of the framing.

Example 6. Let us consider an example of a connected sum where each summand is the result of integral surgery on a knot but the sum is not. Let $\Sigma=$ $L(3,1) \# L(7,1)$. Note that the first homology of $\Sigma$ is cyclic of order 21. Clearly both summands can be obtained by surgery on knots, namely by -3 respectively -7 surgery on the unknot. However $R(\Sigma)=R(L(3,1)+R(L(7,1))=2+6=8$, and as $21 \not \equiv 0 \bmod 8$ we can conclude that $\Sigma$ is not the result of integral surgery on a knot. Note that, as $\Sigma$ can of course be obtained by integral surgery along the trivial two-component link, its surgery number as defined in $[2]$ is two. The same argument shows that all manifolds of the form $L(8 k+3,1) \# L(8 k+7,1)$ have surgery number two.

Example 7. Suppose that $n \equiv 3 \bmod 4$. The lens space $L(n, 1)$ is the result of surgery with framing $-n$ on the unknot. However Corollary 3 shows that this manifold cannot be obtained by integral surgery on a knot with positive framing. In fact, $R(L(n, 1))=n-1, \# H_{1}(L(n, 1) ; \mathbb{Z})=n$, and if $n \equiv 3 \bmod 4$, then $n-1 \not \equiv-(n-1) \bmod 8$. 
As \pm 2 is not a square modulo 5 , Proposition 1 in [8] implies that $L(5,2)$ is not the result of integral surgery on a knot. In [2], D. Auckly gave another argument for this fact which can be generalized to the following

Proposition 4. Assume that $K$ is a knot, $\operatorname{det}(K) \neq \pm 1$, which can be unknotted using only one crossing change, and suppose that neither 2 nor -2 is a square modulo $|\operatorname{det}(K)|$. Let $\Sigma$ denote the double covering of the 3-sphere branched along $K$. Then $\Sigma$ is the result of rational surgery on a knot but cannot be obtained by integral surgery on a knot.

Proof. It is well known that the double branched coverings of two knots which can be obtained from each other by a crossing change are related by rational surgery on a knot, see for instance [2]. As, by assumption, the knot $K$ can be turned into the unknot using one crossing change, it follows that $\Sigma$ is obtained from $S^{3}$ by rational surgery on a knot.

Now suppose for a moment that $\Sigma$ can be obtained as the result of integral surgery on some knot. The trace of this surgery is a simply connected 4-manifold $X$ with second homology $H_{2}(X ; \mathbb{Z})=\mathbb{Z}$. If $n$ denotes the framing of the surgery the intersection form on $X$ is $n$ times the standard form on $\mathbb{Z}$. Using this one easily sees that the linking form on $H_{1}(\Sigma ; \mathbb{Z})$ maps the generator of $H_{1}(\Sigma ; \mathbb{Z})$ to $\pm \frac{1}{|n|}$, see for instance [2] for a proof. Note that $n= \pm \# H_{1}(\Sigma ; \mathbb{Z})= \pm \operatorname{det}(K)$.

It was pointed out in [6] that - as a consequence of the fact that the unknotting number is one -the linking form on $H_{1}(\Sigma ; \mathbb{Z})$ takes precisely the values $k^{2} \frac{2 \epsilon}{|\operatorname{det}(K)|}$ in $\mathbb{Q} / \mathbb{Z}$ for some sign $\epsilon \in\{1,-1\}$, in particular $\frac{2 \epsilon}{|\operatorname{det}(K)|}$ is in the image. Hence there exist integers $a, b \in \mathbb{Z}$ such that

$$
\pm a^{2} \frac{1}{|\operatorname{det}(K)|}=b+\frac{2 \epsilon}{\mid \operatorname{det}(K \mid} \text {. }
$$

Multiplying this by $|\operatorname{det}(K)|$ leads to $\pm a^{2} \equiv 2 \bmod |\operatorname{det}(K)|$ in contradiction to our assumption.

Example 8. Let $K$ denote the Montesinos knot $\mathfrak{m}(1 ;(2,1),(3,2),(3,2))$ which is denoted by $\boldsymbol{8}_{21}$ in [16]. The double covering $\Sigma$ of $S^{3}$ branched along $K$ is then a Seifert fibred space $M(1 ;(2,1),(3,2),(3,2))$, note that this 3 -manifold is actually irreducible. One easily sees that $|\operatorname{det}(K)|=15$ and $u(K)=1$ (the reason being that the rational tangle $t(3,2)$ can be changed to the trivial tangle by one crossing change). As \pm 2 is not a square modulo 15, Proposition 4 applies and we obtain that $\Sigma$ cannot be obtained by integral surgery on a knot although it is the result of rational Dehn surgery on some knot in $S^{3}$. Note that $\# H_{1}(\Sigma ; \mathbb{Z})=15$ and $\sigma(K)=2$, hence $\# H_{1}(\Sigma ; \mathbb{Z})-1 \equiv-R(\Sigma) \bmod 8$, so Corollary 3 does not yield this result.

If a $\mathbb{Z}_{2}$-homology sphere is the result of integral surgery on a link then the 
trace of this surgery will be a natural choice for a 4-manifold bounded by $\Sigma$. Unfortunately this trace will in general not be spin, but one can always do surgery along an embedded surface to obtain a spin manifold.

Lemma 3. Suppose that $W$ is a simply connected 4-manifold whose boundary is a $\mathbb{Z}_{2}$-homology sphere and that $F \subset W \backslash \partial W$ is a closed characteristic surface having non-zero self-intersection number. Let $\epsilon \in\{-1,+1\}$ denote the sign of $F \cdot F$. Then there exists a simply connected spin 4-manifold $X$ with $\partial X=\partial W$ such that

$$
\begin{aligned}
b_{2}(X) & =b_{2}(W)+2(g(F)-1)+|F \cdot F+8 \epsilon \operatorname{Arf}(F)|+4 \operatorname{Arf}(F) \\
\sigma(X) & =\sigma(W)-(F \cdot F+8 \epsilon \operatorname{Arf}(F))
\end{aligned}
$$

where we think of the Arf invariant $\operatorname{Arf}(F) \in \mathbb{Z}_{2}$ as an element of $\{0,1\}$.

Proof. Clearly we can restrict ourselves to the case that the self-intersection number of $F$ is positive, the case of negative self-intersection number then follows by reversing the orientation.

Let us first consider the case that the $\operatorname{Arf}$ invariant $\operatorname{Arf}(F)$ is zero. Let $W^{\prime}$ denote the manifold which is obtained from $W$ by attaching $F \cdot F-1$ copies of $\overline{\mathbb{C} P^{2}}$ and consider the surface $F^{\prime} \subset W$ given by gluing $F$ with the exceptional divisors. Then $F^{\prime} \cdot F^{\prime}=1$, and clearly $\operatorname{Arf}\left(F^{\prime}\right)=\operatorname{Arf}(F)=0$. As explained in [26] we can now construct a sphere $S \in W^{\prime \prime}$, where $W^{\prime \prime}$ is obtained from $W$ by attaching $g(F)$ copies of $S^{2} \times S^{2}$ such that $S \cdot S=F^{\prime} \cdot F^{\prime}=1$ and such that $S$ is still characteristic. Blowing down this sphere gives a spin 4-manifold $X$ as required.

In the case that $\operatorname{Arf}(F)=1$, consider $W^{\prime}=W \# \mathbb{C} P^{2}$ and the surface $F^{\prime} \subset$ $W^{\prime}$ obtained by gluing $F$ with a torus representing 3 times the generator of $H_{2}\left(\mathbb{C} P^{2} ; \mathbb{Z}\right)$. Then $\operatorname{Arf}\left(F^{\prime}\right)=0$ and the claim follows from what we just proved.

Proposition 5. Suppose that a $\mathbb{Z}_{2}$-homology sphere is obtained by integral surgery with framing $n$ on a knot $K$. Let $\epsilon=\frac{n}{|n|}$ denote the sign of the framing and let

$$
\left.\mu=\frac{1}{8}(n-\epsilon)+R(\Sigma)\right) \bmod 2 \in \mathbb{Z}_{2}
$$

(note that, according to Proposition 3, the number in parentheses is actually a multiple of 8). Finally let $h=\# H_{1}(\Sigma ; \mathbb{Z})$ and let $g^{*}=g^{*}(K)$ denote the slice genus of the knot $K$.

(1) If $\mu=0$, then

$$
\frac{4-5 \epsilon}{4}(h-1)+2 g^{*} \geq \bar{m}(\Sigma) \geq m(\Sigma) \geq \frac{-4-5 \epsilon}{4}(h-1)-2 g^{*} .
$$

(2) If $\mu=1$, then

$$
\frac{4-5 \epsilon}{4}(h+7)+2 g^{*}+4 \geq \bar{m}(\Sigma) \geq m(\Sigma) \geq \frac{-4-5 \epsilon}{4}(h+7)-2 g^{*}-4 .
$$


Proof. By assumption, $\Sigma$ is obtained by integral surgery on a knot $K$. The trace of this surgery is a 4-dimensional handlebody $W$ with boundary $\Sigma$ which is the result of attaching a 2-handle along $K$ to $D^{4}$. Consequently $b_{2}(W)=1$ and $\sigma(W)=\epsilon$. By Proposition $3, \operatorname{Arf}(K)=\mu$, and clearly $|n|=h$, i.e. $n=\epsilon h$.

Now pick a surface $F \subset D^{4}$ with boundary $4 \partial F=K$ such that $g(F)=$ $g^{*}(K)$ and let $F^{\prime}$ denote a Seifert surface for $K$. Then the surfaces $G$ and $G^{\prime}$ formed by gluing the core of the 2-handle with $F$ respectively $F^{\prime}$ clearly have the same homology class $\alpha$. As in the proof of Proposition 3, this homology class is characteristic, and $\alpha \cdot \alpha=n$. It is known that the Arf invariant of the surface $G$ obtained by gluing $F^{\prime}$ and the core of the 2-handle is $\operatorname{Arf}(K)$, and as $[G]=\left[G^{\prime}\right]$, the same is true for $G^{\prime}$ Now we can apply Lemma 3 to $W$ to obtain a spin 4-manifold $X$ with boundary $\Sigma$ and the claimed estimates follow from the definitions of the invariants $m$ and $\bar{m}$.

Remark 5. A similar estimate can be derived in the more general case that the $\mathbb{Z}_{2}$-homology sphere $\Sigma$ is obtained by integral surgery on a link which has a characteristic knot (which one can always assume after sliding handles), such a characteristic knot will again define a characteristic surface to which one can apply Lemma 3.

We are now ready to use Proposition 5, combined with the information obtained from Proposition 3, to derive a lower bound for the slice genus of a knot on which integral surgery can be performed to obtain a given $\mathbb{Z}_{2}$-homology sphere $\Sigma$. Note that if $\Sigma$ is the result of integral surgery of a knot, we can, by Corollary 3, choose the orientation of $\Sigma$ such that $\# H_{1}(\Sigma ; \mathbb{Z})-1 \equiv-R(\Sigma) \bmod 8$, therefore we state our result only in this case.

Theorem 2. Assume that $\Sigma$ is a $\mathbb{Z}_{2}$-homology sphere and that $R(\Sigma) \not \equiv 4 \bmod 8$ and $\# H_{1}(\Sigma ; \mathbb{Z})-1 \equiv-R(\Sigma) \bmod 8$. Let

$$
\mu=\frac{1}{8}\left(\# H_{1}(\Sigma ; \mathbb{Z})-1+R(\Sigma)\right) \bmod 2
$$

were we think of $\mu$ as an element of $\mathbb{Z}_{2}=\{0,1\}$. If $\Sigma$ can be obtained by integral surgery on a knot $K$, then

$$
g^{*}(K) \geq \frac{1}{8}\left(\# H_{1}(\Sigma ; \mathbb{Z})-1+4 m(\Sigma)\right)-\mu .
$$

Proof. Let $n$ denote the framing of the knot. Of course $n= \pm \# H_{1}(\Sigma ; \mathbb{Z})$. Once we can show that $n$ must be positive the claimed inequality follows from Proposition 5 . So let us assume that $n<0$. Then $n=-\# H_{1}(\Sigma ; \mathbb{Z})$, and by Proposition 3 , we have

$$
-\# H_{1}(\Sigma)+1 \equiv-R(\Sigma) \bmod 8 .
$$

However combining this with the assumption $\# H_{1}(\Sigma ; \mathbb{Z})-1 \equiv-R(\Sigma) \bmod 8$ 
shows that $R(\Sigma) \equiv-R(\Sigma) \bmod 8$. But this is only possible if $R(\Sigma) \equiv 0 \bmod 4$, which contradicts our assumptions.

Example 9. Assume that $k$ is an even number and consider the lens space $L(16 k+$ $7,7 k+3)$. Note that this lens space is - up to orientation $-L\left(4 m n-1,4 n^{2}\right)$ with $n=2 k+1, m=2$. As $n$ and $m$ are coprime, $L(16 k+7,7 k+3)$ can be obtained by integral surgery on a knot by Theorem 1 in [8]. An admissible continuous fractions decomposition is given by

$$
\frac{16 k+7}{7 k+3}=[2,4,-1,-2,1, k,-1]
$$

Using this one can easily derive that $R(L(16 k+7,7 k+3))=2$ and $m(L(16 k+$ $7,7 k+3)) \geq-1.5$. Hence Theorem 2 implies that the slice genus of any knot $K$ on which integral surgery can be performed to obtain $L(16 k+7,7 k+3)$ must be at least $2 k-1$. Of course the slice genus of a knot is at most its genus, and for every knot the crossing number is at least two times the genus (otherwise Seifert's algorithm would produce a Seifert surface of smaller genus), so we also obtain a lower bound for the crossing number.

The most obvious infinite family of lens spaces which can be obtained by integral surgery on a knot is given by the lens spaces $L(n, 1)$. All these spaces can be obtained by surgery on a single knot, namely the unknot (but of course with different framings). Example 9 shows that this situation is not typical and that one actually has to use infinitely many knots (even infinitely many concordance classes of knots) to obtain all the lens spaces which are the result of integral surgery on a knot, i.e. we have the

Corollary 4. For every natural number $N>0$ there exists a lens space $L(p, q)$ which can be obtained by integral surgery on a knot such that every such knot has slice genus at least $N$.

\section{References}

[1] S. Akbulut, A fake compact contractible 4-manifold, J. Differential Geom. 33 (1991), no. $2,335-356$.

[2] D. Auckly, Surgery numbers of 3-manifolds: a hyperbolic example, Geometric topology, AMS/IP Stud. Adv. Math. 2.1, (Athens 1993), 21-34, Amer. Math. Soc., Providence, 1997.

[3] G. Burde, Über das Geschlecht und die Faserbarkeit von Montesinos-Knoten, Abh. Math. Sem. Univ. Hamburg 54 (1984), 199-226.

[4] G. Burde and H. Zieschang, Knots, Walter de Gruyter \& Co., Berlin, 1985.

[5] A. J. Casson and J. L. Harer, Some homology lens spaces which bound rational homology balls, Pacific J. Math. 96, 23-36.

[6] T. D. Cochran and W. B. R. Lickorish, Unknotting information from 4-manifolds, Trans. Amer. Math. Soc. 297 (1986), no. 1, 125-142.

[7] H. Endo, Linear independence of topologically slice knots in the smooth cobordism group, Top. Appl. 63 (1995), 257-262. 
[8] R. Fintushel and R. J. Stern, Constructing lens spaces by surgery on knots, Math. Z. 175 (1980), no. 1, 33-51

[9] R. Fintushel and R. J. Stern, A $\mu$-invariant one homology 3-sphere that bounds an orientable rational ball, Four-manifold theory (Durham, N. H., 1982), 265-268, Contemp. Math. 35, Amer. Math. Soc., Providence, 1984.

[10] M. Freedman, R. Kirby, A geometric proof of Rochlin's theorem, Proc. Sympos. Pure Math. 32 (1978), 85-97.

[11] Y. Fukumoto and M. Furuta, Homology 3-spheres bounding acyclic 4-manifolds, Math. Res. Letters 7 (2000), 757-766.

[12] M. Furuta, Homology cobordism group of homology 3-spheres, Invent. Math. 100 (1990), no. 2, 339-355.

[13] M. Furuta, Monopole equations and the 11/8-conjecture, Preprint, 1995.

[14] R. E. Gompf and A. I. Stipsicz, 4-manifolds and Kirby calculus, Graduate Studies in Mathematics 20, American Mathematical Society, Providence, 1999.

[15] F. Hirzebruch and D. Zagier, The Atiyah-Singer theorem and elementary number theory, Mathematics Lecture Series, No. 3, Publish or Perish, Boston, 1974.

[16] A. Kawauchi, A survey of knot theory, Birkhäuser Verlag, Basel, 1996.

[17] R. C. Kirby, The topology of 4-manifolds, Lecture Notes in Mathematics 1374, Springer Verlag, Berlin, 1989.

[18] E. Looijenga, The smoothing components of a triangle singularity I, Singularities, Proc. Sympos. Pure Math. 40, 173-183, Amer. Math. Soc., Providence, 1983.

[19] K. Murasugi, On a certain numerical invariant of link types, Trans. Amer. Math. Soc. 117 (1965), 387-422.

[20] H. C. Pinkham, Smoothings of the $D_{p q r}$ singularities, $p+q+r=22$, Proc. Sympos. Pure Math. 40, 373-377, Amer. Math. Soc., Providence, 1983.

[21] L. Rudolph, Quasipositivity as an obstruction to sliceness, Bull. Amer. Math. Soc. (N.S.) 29 (1993), no. 1, 51-59.

[22] N. Saveliev, Fukumoto-Furuta invariants of plumbed homology 3-spheres, Preprint, Max Planck Institut für Mathematik, 2000.

[23] N. Saveliev, Dehn surgery along torus knots, Topology Appl. 83 (1998), 193-202.

[24] T. Tanaka, Unknotting numbers of quasipositive knots, Topology Appl. 88 (1998), no. 3, $239-246$.

[25] K. Walker, An extension of Casson's invariant, Annals of Mathematics Studies 126, Princeton University Press, Princeton, 1992.

[26] A. Yasuhara, Connecting Lemmas and Representing Homology Classes of Simply Connected 4-Manifolds, Tokyo J. Math. 19 (1996), 245-261.

Christian Bohr

Yale University

Department of Mathematics

P.O. Box 208283

New Haven

CT 06520-8283

USA

Current address:

Mathematisches Institut

Theresienstr. 39

80333 München

Germany

e-mail: bohr@mathematik.uni-muenchen.de

(Received: May 7, 2001)
Ronnie Lee

Yale University

Department of Mathematics

P.O. Box 208283

New Haven

CT 06520-8283

USA

e-mail: rlee@math.yale.edu 